\title{
Antimalarial drug resistance in the Eastern Mediterranean Region
}

\author{
A.A. Abdel-Hameed ${ }^{1}$
}

SUMMARY Studies done between 1997 and 2003 are reviewed to give an overall picture of antimalarial drug resistance in the Eastern Mediterranean Region of the World Health Organization (WHO). The situation in 8 countries where resistance has been reported is detailed. It has been difficult to abandon chloroquine as first-line treatment even though resistance to it is widespread. Resistance to sulfadoxine-pyrimethamine has also been detected. The spread of resistance could be slowed down by the adoption of effective national policies and control programmes. Coordination between counties and with other WHO regions, as opposed to working in isolation, could further reduce the spread of resistance.

\section{Introduction}

This review was done to fulfil the following objectives:

- to present a brief overview on the current global concepts concerning antimalarial drug resistance;

- to review historical data about the emergence of drug resistance in the countries of the Eastern Mediterranean Region;

- to present the available information on the current prevalence of drug-resistant falciparum malaria in the Region;

- to set recommendations for measures to prevent the emergence and spread of antimalarial drug resistance in the Region.

This review was based on sources of the following types:

- published in vivo and in vitro studies carried out on antimalarial drug resistance in the countries of the Eastern Mediterranean Region identified through digital databases (Medline ${ }^{\circledR}$ and CAB Health ${ }^{\circledR}$ );
- non-digital publications identified by cross-referencing of the above publications;

- unpublished reports by ministries of health;

- regional and international conference proceedings;

- reports of WHO consultants;

- postgraduate theses;

- personal contacts.

Studies done in 1997-2003 according to recent WHO protocols were selected to describe the current status of drug resistance in the Region.

\section{An overview of drug resistance in malaria}

\section{Definition}

Drug resistance in malaria has been defined as the "ability of a parasite strain to survive and/or multiply despite the administration and absorption of a drug given in doses equal to or higher than those usually recommended but within the tolerance of the

${ }^{1}$ College of Medicine, King Saud University, Riyadh, Saudi Arabia.

المجلة الصحية لشرق المتوسط، منظمة الصحة العالمية، المجلد التاسع، العدد ع، ب... 
subject” [1,2]. Later on a qualification was added to this definition to specify: “... provided that the drug gained access to the parasite or the infected red cell for the duration of time necessary for its action" [3].

Infections due to failure of chemoprophylaxis as has been reported in United States military personnel in Somalia are not considered as treatment failures [4]. Resistance to antimalarial drugs has been described for 2 of the 4 species of human malaria parasites, Plasmodium falciparum and $P$. vivax. Plasmodium falciparum has developed resistance to nearly all antimalarials in current use, although the geographical distribution and prevalence rates of resistance to individual drugs do vary.

\section{Global distribution}

Resistance of $P$. falciparum to chloroquine (CQ) appeared almost simultaneously in Colombia and in south-east Asia in 1960. Today, CQ-resistant falciparum malaria is being reported from all countries in which the disease is endemic except for few foci in central America north of the Panama Canal, Haiti and the Dominican Republic [5]. This created a great problem for malaria control programmes, which depended on the cheap and effective CQ as a first-line treatment. In areas of CQ resistance, sulfadoxine-pyrimethamine was the usual choice as a replacement as first-line treatment for uncomplicated malaria. However, sulfadoxine-pyrimethamine resistance emerged in south-east Asia and south America and is now on the increase in Africa. Mefloquine resistance is frequent in some areas of south-east Asia and has been reported in the Amazon region of south America and sporadically in Africa [6]. Resistance to quinine is often overestimated since the dosage of $24 \mathrm{mg}$ base per $\mathrm{kg}$ for seven days is rarely respected, and the threshold for in vitro resistance has not been clearly defined. Plasmodium vivax infection resistant to $\mathrm{CQ}$ and/or primaquine has a limited distribution $[7,8]$.

\section{Methods of monitoring drug resistance}

In vivo tests

The first standardized test systems for the assessment of in vivo drug response in $P$. falciparum were developed shortly after the first reports of $\mathrm{CQ}$ resistance in this species [1]. These test systems were subsequently revised [9] and have remained basically unchanged since the WHO Scientific Group on the Chemotherapy of Malaria and Resistance to Antimalarials in 1972 put them in their present form [2]. The standardized tests were originally developed for CQ but, with the appropriate modifications, they could be used for the evaluation of the response to other blood schizontocidal drugs. These tests follow set criteria for the selection of patients, the administration of a standard treatment regimen of the appropriate drug, and daily parasitological blood examination for the stipulated period, e.g. 7 or 28 days for CQ, or longer periods for drugs with a long elimination half-life.

In spite of the valuable information so far yielded by these standard tests, their application in the field faced considerable constraints due to the need for daily blood examination, especially when follow-up had to be extended beyond 7 days. In addition, these tests were primarily conceived for the assessment of the parasitological response of $P$. falciparum in non-immune persons, and took practically no note of the clinical response to the drugs beyond the recognition of overt initial failure as a criterion for discontinuing the test. For these reasons, WHO started a scheme to devise a new test system that would be simple and at the same time provide enough informa-

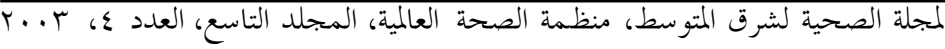


tion for the formulation and updating of malaria treatment policies [10]. Since 1996 a number of versions of the protocol have been drafted, the latest being issued in 2003 [11].

\section{In vitro tests}

In vitro tests measure the intrinsic sensitivity of $P$. falciparum and $P$. vivax to antimalarial drugs, independent of host immunity. They can be used for recognizing changes in drug sensitivity even before the advent of overt in vivo resistance; for assessing baseline response before the introduction of a new drug; for monitoring sensitivity to drugs that cannot be used in monotherapy; for assessing sensitivity to candidate drugs; and for identifying cross-sensitivity and cross-resistance patterns between drugs. In association with in vivo tests, they can be employed for determining the threshold concentration of resistance for specific drugs. A special application relates to the sensitivity monitoring of imported infections. The most commonly used in vitro test system is the standard micro-technique for assessing the inhibition of schizont maturation first published in 1978 [12]. Currently, a revised methodology, the Mark III test, is being used [13].

The results of in vitro tests can be used to determine individual or geometric mean cut-off concentrations of schizont maturation (P. falciparum) or growth ( $P$. vivax). The test results are usually also processed by log concentration-response probit analysis for the determination of the $50 \%, 90 \%$ and 99\% effective concentrations (EC50, EC90 and EC99) and other regression parameters.

\section{Molecular markers}

Molecular markers are very useful in the in vivo test to differentiate between re- infection and recrudescence during the follow-up of the patient by comparing 3 polymorphic genes, MSP1, MSP2 and GLURP. Chloroquine resistance has been associated with changes in one or more of several candidate genes. PfCRT (P. falciparum CQ resistance transporter) has been identified as the potential gene involved in CQ resistance. The P. falciparum multidrug resistance gene 1 (PfMDR 1), which encodes for the P-glycoprotein homologue 1 protein (Pgh1), is thought to increase CQ resistance [14]. Sulfonamides and sulfones act on folate biosynthesis by inhibiting dihydropteroate synthetase (DHPS), whereas biguanides and diaminopyrimidine inhibit dihydrofolate reductase (DHFR). Resistance arises through single point mutations in the plasmodial DHFR and dihydropteroate (DHPS) genes. At present, studies are under way to establish the relationship between antifolate resistance and the relative frequencies of these mutations in different epidemiological settings in order to allow their use as public health surveillance tools to monitor drug resistance [15].

\section{Impact of antimalarial drug resistance}

One of the immediate effects of the emergence of drug resistance in an endemic area could show as increased malaria morbidity and mortality that could reach epidemic proportions, as happened in 1988 in Somalia $[16,17]$. In areas where falciparum and vivax malaria co-exist, the emergence of drug-resistant $P$. falciparum strains could led to a relative increase in the prevalence of this species. This is believed to be why CQ-resistant $P$. falciparum malaria has become more prevalent during the last decade in Pakistan [18], Afghanistan [19] and neighbouring Tajikistan [20]. In the United Arab Emirates imported resistant strains

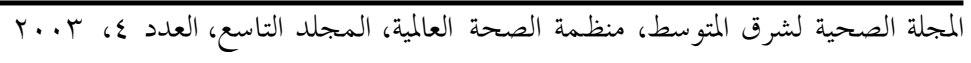


posed the threat of re-introduction of malaria in an area in which the disease had been eliminated [21]. Emergence of drug resistance is known to be associated with increased costs to malaria control programmes because replacement antimalarials are usually more expensive than the original drugs [22]. When an increasing proportion of cases are taking a first-line antimalarial that fails, to be followed by a course of the second-line drug, then there would be a point at which it would be much cheaper to replace the first-line drug by the second-line. Moreover, the total cost of treatment of cases with treatment failure that progress to severe disease requiring hospitalization is much higher than home treatment of an uncomplicated attack [23].

\section{Factors associated with emergence and spread of antimalarial drug resistance}

The emergence and spread of drug resistant malaria is influenced by interactions between the antimalarial drugs, the malaria parasites, humans and the mosquito vectors $[24,25]$. The basic event in the emergence of drug resistance is acquisition by the parasite of the ability to evade drug action. This occurs by different mechanisms for the different antimalarial drugs. Drug pressure leads to selection of resistant strains. When the natural population of malaria parasites is exposed to sub-therapeutic levels of the drug, only highly sensitive parasites are eliminated. If this drug pressure is maintained, moderately sensitive parasites are eliminated, and so on, until we end up with resistant parasites. A variety of factors can lead to the creation of drug pressure, the most important of these being poor patient or prescriber compliance with the standard dose. A number of studies have demonstrated the widespread practice of self-medication and irrational prescribing of antimalarial drugs in Pakistan [26], Somalia (M. Warsame, unpublished report, 1998), Sudan $[27,28]$ and the Republic of Yemen [29].

For the successful chemotherapy of malaria, the effect of drugs is complemented by the body's immune system, which helps in clearance of the parasites. Due to lack of acquired immunity, drug resistance usually manifests first in the non-immune segments of the population. An example of this was the spread of drug resistant malaria among non-immune Eritrean refugees in eastern Sudan [30,31]. In populations with established drug resistance, movement of these populations from one area to another could help to spread resistance, as happened following the movement of Afghan refugees into parts of Pakistan and the Islamic Republic of Iran [18,32].

Antimalarials vary in their post-therapeutic selection span due to variations in their elimination half-life, from a few hours for quinine to 10-40 days for mefloquine. This mechanism causes significant selection pressure in drugs with a long elimination half-life, i.e. drugs that are long-lived at sub-therapeutic levels. It follows that drug resistance develops much easier to mefloquine and sulfadoxine-pyrimethamine, with their long half-life [25].

The net effect of the emergence of drug resistance on the intensity of malaria transmission is not fully understood [33]. In many instances the emergence of drug resistance is associated with increased transmission, irrespective of drug pressure. There are also foci where drug resistance has still failed to be established; this is probably due to poor parasite-vector compatibility.

المجلة الصحية لشرق المتوسط، منظمة الصحة العالمية، المجلد التاسع، العدد ؟، ب.. 


\section{Antimalarial drug resistance in the countries of the Eastern Mediterranean Region}

In the WHO Eastern Mediterranean Region, transmission of $P$. falciparum and drug resistance is being reported in three zones: south Asia, (Afghanistan, Islamic Republic of Iran and Pakistan), Africa south of the Sahara (Djibouti, Somalia and Sudan) and the Arabian peninsula (Saudi Arabia and Republic of Yemen).

\section{Afghanistan}

Malaria has a patchy distribution throughout Afghanistan except for the central highlands, which are malaria-free above an altitude of 1500 metres. The disease is endemic in the lowlands, especially the rice growing areas, but it also occurs in urban areas such as Kabul and Jalalabad. The predominant species is $P$. vivax, accounting for $80 \%$ to $90 \%$ of cases. Chloroquine is recommended in standard doses as a firstline drug for treatment of uncomplicated infections with $P$. falciparum and $P$. vivax. However, due to reports of CQ resistance, sulfadoxine-pyrimethamine has also been recommended as a first-line drug for uncomplicated falciparum malaria in Afghanistan. The first case of drug resistant falciparum malaria occurred in 1986 [34].

Chloroquine-resistant $P$. falciparum has been reported among Afghan refugees in Pakistan [18,35] and in Iran [36]. Most (90\%) of the cases of $P$. falciparum malaria among the Afghan refugees in western Pakistan were sensitive to sulfadoxinepyrimethamine [35]. A study conducted in Afghanistan in 1999 showed 67\% P. falciparum infection resistant to CQ in the eastern part of the country, with $11 \%$ having RII-RIII resistance [19]. All cases were successfully treated with sulfadoxinepyrimethamine.

\section{Djibouti}

Since 1978 malaria has become endemic in Djibouti. The epidemiological situation is characterized by malaria outbreaks that have a particularly serious impact on the most vulnerable groups. The absence of epidemiological surveillance and coordination of public services renders the control of the epidemic difficult and encourages the development of resistance to 4-aminoquinolines in $P$. falciparum (the prevailing parasite species). There are no recent data on the therapeutic efficacy of drugs against malaria parasites in Djibouti. Although cases of treatment failure following CQ have been noted, the presence and extent of resistance needs to be confirmed. The last in vitro tests were done in 1990 by a team from the US Naval Medical Research Unit in Cairo (NAMRU-3) in collaboration with health service staff in Djibouti in order to test the sensitivity of $P$. falciparum to CQ. The standard in vitro micro-test was used. In total, $21 P$. falciparum isolates were tested, 10 were classified as sensitive, 9 were classified as borderline and two isolates were classified as resistant. There is definitely a need for an updated assessment of the status of resistance using standard methodology [37].

\section{Islamic Republic of Iran}

Malaria-endemic areas of the Islamic Republic of Iran are located in the south-eastern part of the country, between the coast and Pakistan to the east. These areas include the provinces of Sistan-Baluchestan, Hormozgan and Kerman (mainly the Kahnoudj district). The predominant species is $P$. vivax, accounting for about $90 \%$ of infections, the rest being due to $P$. falciparum. The recommended first-line antimalarial treatment is CQ, with sulfadoxine-pyrimethamine recommended as the second-line treatment for uncomplicated

المجلة الصحية لشرق المتوسط، منظمة الصحة العالمية، المجلد التاسع، العدد ؟، ب... 
cases. It is believed that drug resistant strains of malaria parasites might have been introduced by the influx of refugees from Afghanistan in recent years [34].

Resistance of $P$. falciparum to CQ was first observed in Iranshahr in SistanBaluchestan province in 1983 [38] and later in Bandar-e Abbas, Hormozgan province, both situated in the south-east [39].

The response to antimalarial drugs has been evaluated for a large number of cases referred to the malaria laboratories at Bandar-e Abbas and Iranshahr health research and training centres during the period 1990-1996 [36]. Enrolled cases were referred from malaria out-patient clinics in Bandar-e Abbas. These studies used the standard WHO extended in vivo test [40] and Mark II in vitro test [41]. In the first phase of the study (1990-93), 593 falciparum cases $(73.4 \%)$ were resistant at RI (45.5\%), RII (16.7\%) and RIII (11.2\%) levels. Of 88 CQ-resistant patients treated with sulfadoxine-pyrimethamine, 12 cases (13.6\%) were also resistant to sulfadoxinepyrimethamine at RI (6.8\%) and RII (6.8\%) levels. In the in vitro test, 1011 blood samples were collected from falciparum malaria patients and examined against CQ, amodiaquine, sulfadoxinepyrimethamine, mefloquine and quinine. Among 508 cases in which the growth of the parasite was successful, the resistance rates to the drugs were $33.4 \%, 15.2 \%$, $17.9 \%, 2.2 \%$ and $0.0 \%$, respectively. In the second phase of the study (1994-96), 297 cases (60.2\%) were resistant to CQ at RI (46.9\%), RII (10.5\%) and RIII (2.8\%) levels. Parasites were totally sensitive in vitro to mefloquine in 33 samples taken from patients from the Iranshahr area during 1990-1993. In most cases, no growth was observed in any mefloquine treated well. However, sporadic cases of innate re- sistance to mefloquine in vitro had previously been reported in Iran [42,43].

Response to antimalarial drugs continued to be similarly monitored in south-eastern areas at two sentinel sites, with results for 2001 indicating resistance to CQ at RI of $65.2 \%$, RII $6.5 \%$ and no cases at RIII [37]. Although all the samples tested for sulfadoxine-pyrimethamine at these sites in 2001 were fully sensitive to the drug, preliminary molecular studies done in this area of the country have revealed a high prevalence of a DHFR mutation associated with sulfadoxine-pyrimethamine resistance $[44,45]$. This highlights the need for close monitoring for the emergence of resistance to this antimalarial.

\section{Pakistan}

Malaria in Pakistan belongs to the oriental eco-epidemiological type. About $40 \%$ of cases are due to $P$. falciparum, which is significantly more common in Sindh province (64\%). Pakistan was the first country in this region to establish a system for monitoring the efficacy of antimalarial drugs in 1997 (S.M. Mujtaba, I.H. Shah, M.A. Shah, unpublished report, 1981), prompted by concern over the emergence of resistant parasite strains in India. The programme concentrated on field studies to assess the response of $P$. falciparum to CQ, mainly by using the classical WHO extended test [2]. The first cases of CQ resistant falciparum malaria in Pakistan were reported in 1984 [46,47]. Data from these studies were reviewed by Shah et al. [18]. Most of the tests were done in Punjab province, involving small numbers of cases in each survey. Variable rates of RI and RII resistance were detected but no cases of RIII were reported. The past decade has witnessed a resurgence of falciparum malaria and the spread of resistant strains has been

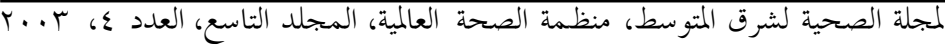


reported. However, recent information regarding drug resistant malaria in Pakistan remains incomplete. In 1998 the Eastern Mediterranean Regional Office of WHO undertook the re-establishment of sentinel sites in Pakistan for monitoring the therapeutic efficacy of antimalarial drugs using standard WHO protocols (A.A. Adeel, unpublished report, 1998).

\section{Saudi Arabia}

In Saudi Arabia the prevalence of malaria has been on the decrease over the past few years. Currently, there are two main malaria endemic areas in the country, in the south (Asir) and the south-west (Jizan). There are a number of case reports of CQ resistance [48-54] but the main evidence comes from the annual random screening conducted by the Ministry of Health. Results of this screening indicate that the endemic $P$. falciparum strains that are resistant to CQ by in vitro test steadily increased in the Jizan region between 1986 and 1998 [37]. The therapeutic efficacy of CQ against $P$. falciparum has recently been assessed by Ghalib et al., who reported a treatment failure rate of $12 \%$, which is probably an underestimate [55]. It should be noted that the protocol they used did not conform to standard WHO methodology. Patients with any degree of parasitaemia on day 3 were considered as treatment failure and given alternative treatment.

Even in the other Gulf States like Kuwait and the United Arab Emirates, where there is no active malaria transmission, malaria is still a matter of concern since a significant proportion of imported cases seen in clinical practice are drug resistant infections [56,57].

\section{Somalia}

In Somalia there are different epidemiological strata of malaria transmission, resulting in varying grades of population immunity and consequent variable response to antimalarials. There are areas of intense transmission along the two rivers (Shebele and Jubba) and in the south and central regions and there are also hypoendemic areas of unstable transmission in the north-west and north-east. The predominant species of malaria is $P$. falciparum. There is no official national malaria treatment policy in Somalia. Both CQ and sulfadoxine-pyrimethamine are widely used for first-line treatment. Self-medication and irrational use of antimalarials are common [17].

The first published indications of the emergence of drug-resistant malaria in Somalia were from Ethiopia, where the first cases of CQ-resistant falciparum malaria were found to be confined to areas of the borders with Somalia, Sudan and Kenya [58]. Cases on the Ethiopia-Somalia borders were classified at RII/RIII based on the WHO 28-day test (the extended test). The Ethiopia-Somalia border areas are porous boundaries, frequently crossed by refugees, traders and immigrants and predominantly inhabited by nomads who move freely in search of grazing pastures. At about the same time, in a field study carried out in Malable, $132 \mathrm{~km}$ south of Mogadishu, the WHO 7-day in vivo test resulted in parasite clearance in all patients within 7 days (S/RI) while the in vitro test showed a low level of resistance in 3 out of 29 samples tested [59].

Subsequent studies indicated a rapid spread and intensification of CQ resistance in Somalia. Over the 4 years that the Malable area was monitored the prevalence of cases classified as RII/RIII rose from $17 \%$ in 1987 to $19 \%$ in 1988 and $30 \%$ in 1989 [60]. In the same study, the in vitro test demonstrated the steady rise of CQ resistance as the proportion of resistant isolates rose from 3/29 in 1986 to $12 / 19$ in 1989.

المجلة الصحية لشرق المتوسط، منظمة الصحة العالمية، المجلد التاسع، العدد ؟، ب... 
There was also a rise in the mean effective dose of CQ, EC50 (50\% effective concentration) and EC99, rising from $0.34 \times 10^{-6}$ $\mathrm{mol} / \mathrm{L}$ and $1.99 \times 10^{-6} \mathrm{~mol} / \mathrm{L}$ respectively in 1986 to $0.78 \times 10^{-6} \mathrm{~mol} / \mathrm{L}$ and $7.5 \times 10^{-6}$ $\mathrm{mol} / \mathrm{L}$ respectively in 1989 . The emergence and spread of CQ resistance was closely associated with the malaria epidemic that occurred in Balcad in 1988 [16,17]. In a hospital sample investigated during this epidemic the in vivo response of $P$. falciparum to the standard therapeutic regimen of CQ showed a high degree of resistance, with 31 of 36 patients showing resistance, including 5 RI responses and 26 RII/RIII responses. Micro in vitro tests for CQsusceptibility showed resistance in 33 out of 37 isolates with a mean EC50 and EC99 of $1.50 \times 10^{-6} \mathrm{~mol} / \mathrm{L}$ and $10.97 \times 10^{-6} \mathrm{~mol} /$ $\mathrm{L}$ respectively, indicative of a high degree of CQ resistance. After the onset of civil strife in 1991, the situation deteriorated further due to deterioration of the health infrastructure and mass population movements.

Starting from 1997, studies done in Somalia to assess the response of falciparum malaria to antimalarials adopted the newer approach, aiming at assessment of clinical as well as parasitological response to chemotherapy [61]. With this approach, Warsame et al. investigated the response of falciparum malaria to treatment at 2 sites, at Merca, an area of intense transmission, and at Gabiley, an area of low transmission [62]. Treatment with CQ resulted in clinical failure in 33\% $(n=60)$ and 51\% $(n$ = 49) of the patients in Merca and Gabiley respectively. There were corresponding parasitological failures of $77 \%$ RII/RIII and 35\% RII/RIII. With the sulfadoxinepyrimethamine treatment, 98\% $(n=50)$ of the patients achieved an adequate clinical response despite a parasitological failure rate of $76 \% \mathrm{RII} / \mathrm{RIII}$. It was concluded that CQ should no longer be considered ad- equate for treating clinical falciparum malaria in vulnerable groups in the areas studied. Doubts about the therapeutic life of sulfadoxine-pyrimethamine in relation to malaria were raised by the high levels of resistance in the Merca area, underlining the need to identify suitable alternatives.

Although previous studies had indicated the efficacy of mefloquine against $P$ falciparum malaria in Somalia [63], 5 cases of mefloquine prophylaxis failure were reported among 41 cases of military personnel of the United States of America stationed in Somalia 1992-93. All 5 cases had both serum levels $\geq 650 \mathrm{ng} / \mathrm{mL}$ and metabolite:mefloquine ratios $>2$, indicating chemoprophylactic failure. It has also been reported that primaquine therapy was ineffective in preventing relapses in cases of vivax malaria acquired in Somalia, suggesting the presence of primaquine-resistant $P$. vivax strains [64].

Currently, work is under way to establish a number of sentinel sites to cover different epidemiological zones in the country using standard WHO protocols [11]. Two sites, Jamaame, in the south of the country, and Jawhar, $90 \mathrm{~km}$ north of Mogadishu, are now operational, and preliminary results indicate high levels of early treatment failure of CQ and parasitological failure of sulfadoxine-pyrimethamine [65].

\section{Sudan}

There are different epidemiological strata of malaria in Sudan, leading to different levels of community immunity to malaria, and this affects therapeutic response to treatment. In southern Sudan malaria is holoendemic in the equatorial and rich savannah belt and hyperendemic in the savannah zone. In central Sudan it is mesoendemic in the poor savannah and semi-desert zones. In northern Sudan malaria is hypoendemic along the Nile and along the Red Sea hills.

المجلة الصحية لشرق المتوسط، منظمة الصحة العالمية، المجلد التاسع، العدد ع، ب...T 
Transmission ranges from perennial in the south to seasonal in the other parts of the country.

Up to 1970 , CQ-resistant $P$. falciparum had not been confirmed in Africa [66]. The drug was cheap, effective and tolerable. It is widely believed that the first confirmed cases of CQ-resistant falciparum malaria were those of the American tourists returning from Kenya in 1979. On reporting the cases, Kean made a very dramatic statement, "All malariologists have dreaded the day when chloroquine-resistant malaria from Africa would be described. Alas, evidence presented in two case reports would indicate that the day is here" [67].

By that time, CQ-resistant falciparum malaria had already been suspected and confirmed in a small number of indigenous cases in the Sudan. This had been done in an in vivo study carried out in 1978, using the WHO standard in vivo test for 28 days in Gezira and for 7 days in Bor [68]. Three cases of resistance were identified in Gezira but no cases were detected in Bor. Because the follow-up was done for only 7 days in the Bor area, it was possible that cases with RI resistance could have been missed. In the same year a team from WHO conducted in vitro and in vivo studies in Sennar and reported that $P$. falciparum had lower sensitivity to CQ by the in vitro test than other African strains, and that parasite clearance time was slower by the in vivo test than in other African cases of malaria tested [69].

A definite confirmation of the extent of CQ resistance in Sudan was first demonstrated by an in vivo study in the Khartoum area. The study was done in April 1982 in 8 villages about $25 \mathrm{~km}$ north of Khartoum. The test was carried out in 26 patients aged 4-20 years who were observed for 28 days. Ten were sensitive, 9 had RI and 7 had RII responses. It was also noted that parasite clearance was slower and more incomplete than in the previous 2 studies conducted in Sudan [70,71].

In the 1980s eastern Sudan faced a number of disasters, including drought, famine, floods, war and influx of refugees from Ethiopia. Under these conditions malaria outbreaks were frequent, with reports of substantial numbers of cases not responding to CQ.

A study combining in vivo and in vitro assessment done in 1986 in eastern Sudan indicated $43 \%$ of $P$. falciparum infections investigated were resistant at RI, RII or RIII levels [30]. Subsequently, clinical and laboratory reports indicated that resistant $P$. falciparum was widespread in Sudan [31,32,72-80] (E. Stivanello, unpublished report, 2003).

It is difficult to compare the earlier data because different methods were used, but a good number of these studies did conform to the standard in vivo and in vitro tests developed by WHO. Application of these tests to cover the whole country on a periodic basis met with several difficulties due to the complicated nature of the tests and the difficult field conditions. In 1997 a plan was launched by the Federal Ministry of Health and WHO to establish sentinel sites to cover the different epidemiological strata in the country.

As a result of this effort, work was initiated in a number of sentinel sites. This was intended to be a continuous process whereby the country would be covered in time and space by a system of monitoring that would be the source of a continuous flow of information on the therapeutic efficacy status of antimalarials. Work in most of these sites was discontinued, however, after one round of the test owing to problems faced in these sites such as lack of training, lack of close supportive supervision, unstable staff and poor incentives for

المجلة الصحية لشرق المتوسط، منظمة الصحة العالمية، المجلد التاسع، العدد ؟، ب... 
the workers. In spite of these problems, the programme did provide valuable information from its inception. The programme has been recently reactivated and another training workshop for field workers was held in 2002. Data from these sites and from other independent studies indicate a high level of resistance to CQ and a high likelihood of the development of resistance to sulfadoxine-pyrimethamine.

In all, 26 studies were done based on WHO protocols [37,73-75] (I.A. Ahmed et al., unpublished report) (E. Stivanello, unpublished report, 2003). These studies covered 17 different sentinel sites in 11 states. Chloroquine was assessed in 25 tests, of which 22 indicated a prevalence rate of drug resistance at or greater than 25\%. Sulfadoxine-pyrimethamine was tested in southern Sudan and treatment failure rates were reported to be $69.9 \%$ (E. Stivanello, unpublished report, 2003), whereas a study in Khartoum in northern Sudan reported $11.69 \%$ of tested cases classified as RI or RII [75].

Besides the evidence from field studies, there are a number of clinical case reports on Sudanese patients diagnosed with CQresistant malaria after they travelled abroad to areas with no natural malaria transmission [56,57].

A number of molecular studies on Sudanese isolates of $P$. falciparum have led to a better understanding of the molecular basis of drug resistance [81-84].

\section{Republic of Yemen}

There are 4 major epidemiological strata of malaria in the Republic of Yemen. These are coastal plain, foothills, mountains and the island of Sokotra. Malaria-free zones include the mountain plateau and arid slopes from the highlands to the desert. The population at risk of malaria constitutes $60 \%$ of the total population, with an estimated 3 million cases a year and a mortality rate estimated to be $1 \%$ of cases. The predominant species is $P$. falciparum.

Most of the studies on antimalarial drug efficacy carried out in the past 2 decades were done in the southern parts of the country (D.K. Berga, unpublished report, 1999). These were mainly in vivo studies based on the standard WHO 7-day test. A study conducted in Al Makatra (Ta'izz governorate) in 1987 demonstrated the presence of CQ-resistant parasites. In this study, 3 of 50 patients included in the study failed to clear asexual parasitaemia during the 28-day follow-up period. Only low (RI) level resistance was demonstrated in all these patients. Eight isolates were found resistant to CQ out of 72 isolates tested in vitro in Hodeida and Ta'izz in 1989. Since then, the status of CQ sensitivity was determined only sporadically until 1993. During the period 1993-1998 no survey was carried out on drug resistance by the malaria control programme in the country. Most of the studies were carried out in the late 1980s and early 1990s by WHO consultants for malaria control. From the national side, mainly laboratory technicians were involved in the studies. More recently, an in vivo drug resistance study was conducted in Ibn Khaldun Hospital (Lahij governorate) in May 1998. Of the 100 patients included in this study, resistance was detected in 9 patients, 2 moderate (RII) and 7 low grade (RI) resistance levels. Patients were admitted to the hospital for 1 week and followed up weekly for 28 days after discharge.

A national project for establishing sentinel sites for monitoring the therapeutic efficacy of antimalarial drugs to $P$. falciparum based on the standard WHO protocol has now been established (A.A. Adeel, unpublished report, 2002). Three sentinel sites are now operational, in Bajil (Hodeida governorate), Musemir (Lahij governorate) and

المجلة الصحية لشرق المتوسط، منظمة الصحة العالمية، المجلد التاسع، العدد ؟، ب..r 
Udayn (Ibb governorate). The first round of tests assessed the response of falciparum malaria to CQ. Preliminary results from the 3 sites indicate high levels of treatment failure with CQ (M. Khalifa, personal communication).

\section{Problems and constraints}

In all the countries reviewed, CQ remains the first-line treatment for uncomplicated malaria. In spite of the emergence and spread of CQ resistance to significantly high levels in some areas, it has been difficult to abandon this drug and move to an alternative second-line drug. A delay in changing the first-line drug would result in excess, avoidable morbidity and mortality [10]. When we postulate changing treatment practices to accommodate changes in drug susceptibility, we find problems at 3 different levels. First, there are problems in gathering and presenting evidence on drug resistance. The second level is that of articulating this evidence into new policies and the third level is changing policies into practice.

\section{Conclusions and recommendations}

- Drug-resistant falciparum malaria poses a problem in all countries of the WHO Eastern Mediterranean Region, especially those in which the disease is endemic.

- The emergence of resistance to antimalarial drugs is inevitable, but could be slowed down. An important approach to controlling the emergence and spread of drug resistance is to limit drug pres- sure. This could be achieved through an effective antimalarial drug policy, improvement in prescription practices of health workers and improving patient compliance.

- A number of countries within the Region share the same problem because the continuous population movements between these countries make drug resistance a common threat for their malaria control programmes. It would be more effective if such problems were tackled by integrating efforts between these countries rather than working in isolation. The recent "Intercountry workshop on monitoring therapeutic efficacy of antimalarial drugs", held in Sana'a, Republic of Yemen 21-25 April 2002 made a significant contribution towards this end by having the plans of action for the Region and for each country on the table for discussion. Inter-regional links should also be encouraged to promote coordination of efforts with neighbouring countries that belong to other WHO regions.

- To reduce the need for drugs, other measures for control of malaria transmission, such as insecticide-treated materials, should be supported. To limit the spread of resistant malaria strains, it would be highly desirable to use gametocytocidal drugs.

- The possibility of introducing drug combinations should be investigated in terms of financial feasibility and possible impact on malaria case management and the malaria situation in general. This could be facilitated by operational research on the different aspects pertaining to a change of the first-line drug for malaria. 


\section{References}

1. Resistance of malaria parasites to drugs. Geneva, World Health Organization, 1965 (WHO Technical Report Series, No. 296).

2. Chemotherapy of malaria and resistance to antimalarials. Geneva, World Health Organization, 1973 (WHO Technical Report Series, No. 529).

3. Bruce-Chwatt LJ et al. Chemotherapy of malaria, 2nd rev. ed. Geneva, World Health Organization, 1986 (WHO Monograph Series, No.27).

4. Wallace MR et al. Malaria among United States troops in Somalia. American journal of tropical medicine and hygiene, 1996, 100(1):49-55.

5. Bloland PB. Drug resistance in malaria. Geneva, World Health Organization, 2001 (WHO/CDS/CSR/DRS/2001/4).

6. Mockenhaupt FP. Mefloquine resistance in Plasmodium falciparum. Parasitology today, 1995, 11:248-53.

7. Murphy GS et al. Vivax malaria resistant to treatment and prophylaxis with chloroquine. Lancet, 1993, 341(8837):96-100.

8. Looareesuwan $S$ et al. Primaquinetolerant vivax malaria in Thailand. Annals of tropical medicine and parasitology, 1997, 91:939-943.

9. Chemotherapy of malaria. Geneva, World Health Organization, 1967 (WHO Technical Report Series, No. 375).

10. Antimalarial drug policies: Data requirements, treatment of uncomplicated malaria and management of malaria in pregnancy. Report of an informal consultation. Geneva 14-18 March 1994. Geneva, World Health Organization, 1994 (unpublished document WHO/ $\mathrm{MAL} / 94.1070$, available on request from Communicable Disease Prevention and
Control, World Health Organization, 1211 Geneva 27, Switzerland).

11. Assessment and monitoring of antimalarial drug efficacy for the treatment of uncomplicated falciparum malaria. Geneva, World Health Organization, 2003 (WHO/HTM/RBM/2003.50).

12. Rieckmann $\mathrm{KH}$ et al. Drug sensitivity of Plasmodium falciparum. An in-vitro microtechnique. Lancet 1978, 1(8054):223.

13. In vitro micro-test (Mark III) for the assessment of the response of Plasmodium falciparum to chloroquine, mefloquine, quinine, amodiaquine, sulfadoxine/pyrimethamine and artemisinin. Instructions for use of the in vitro micro-test kit (Mark III). Geneva, World Health Organization, 2001 (CTD/MAL/ 97.20 Rev.2).

14. Djimde $A$ et al. A molecular marker for chloroquine-resistant falciparum malaria. New England journal of medicine, 2001, 344(4):257-63.

15. Kublin JG et al. Molecular markers for failure of sulfadoxine-pyrimethamine and chlorproguanil-dapsone treatment of Plasmodium falciparum malaria. Journal of infectious diseases, 2002, 185(3):380-8.

16. Warsame $M$ et al. Isolated malaria outbreak in Somalia. Role of chloroquine resistant Plasmodium falciparum demonstrated in Balcad epidemic. Journal of tropical medicine and hygiene, 1990, 93(4):284-9.

17. Warsame M et al. An epidemic of Plasmodium falciparum malaria in Balcad, Somalia, and its causation. Transactions of the Royal Society of Tropical Medicine and Hygiene, 1995, 89:142-5.

المجلة الصحية لشرق المتوسط، منظمة الصحة العالمية، المجلد التاسع، العدد ؟، ب... 
18. Shah I et al. Chloroquine resistance in Pakistan and the upsurge of falciparum malaria in Pakistani and Afghan refugee populations. Annals of tropical medicine and parasitology, 1997, 91(6):591-602.

19. Rab MA et al. Resistance of Plasmodium falciparum malaria to chloroquine is widespread in eastern Afghanistan. Annals of tropical medicine and parasitology, 2001, 95(1):41-6.

20. Pitt $S$ et al. War in Tajikistan and reemergence of Plasmodium falciparum. Lancet, 1998, 352(9136):1279.

21. Dar FK et al. Status of imported malaria in a control zone of the United Arab Emirates bordering an area of unstable malaria. Transactions of the Royal Society of Tropical Medicine and Hygiene, 1993, 87(6):617-9.

22. Practical chemotherapy of malaria. Report of a WHO Scientific Group. Geneva, World Health Organization, 1990 (WHO Technical Report Series, No. 805).

23. Abdel-Hameed AA, Abdalla HM, Alnaury $\mathrm{AH}$. Household expenditure on malaria case management in Wad-Medani, Sudan. African journal of medicine and medical sciences, 2001, 30(suppl.):358.

24. Wernsdorfer WH. The development and spread of drug resistant malaria. Parasitology today, 1991, 7:297-303.

25. Wernsdorfer WH. Epidemiology of drug resistance in malaria. Acta tropica, 1994, 56:143-56.

26. Shah MA, Ahmad Z. Malaria trends in general practice in Karachi, Pakistan. Journal of the College of Physicians and Surgeons-Pakistan, 1996, 6:62-4.

27. Abdel-Hameed AA. Malaria case management at the community level in Gezira, Sudan. African journal of medicine and medical sciences, 2001, 30(suppl.):43-6.
28. Yousif MA, Adeel AA. Antimalarial prescribing patterns in Gezira State: precepts and practices. Eastern Mediterranean health journal, 2000, 6(5-6): 939-47.

29. Abdo-Rabbo A. Rational prescribing of antimalarial drugs. Unpublished paper presented at the Intercountry Workshop on Monitoring Therapeutic Efficacy of Antimalarial Drugs, Sanaa, Republic of Yemen 21-25 April 2002. Available at http://www.emro.who.int/rbm/meetings/ yemen02/Presentations/SUNDAY/ WHO-PRESCR.RATION.OF\%20 ANTIMALAR-Presentation.doc(a c ces sed 19/05/2004).

30. Bayoumi RA et al. Chloroquine resistant Plasmodium falciparum in eastern Sudan. Acta tropica, 1989, 46(3):15765.

31. Saeed BO et al. Plasma chloroquine measurement in the evaluation of Plasmodium falciparum sensitivity. Journal of tropical medicine and hygiene, 1990, 93:(6):393-6.

32. Edrissian $\mathrm{GH}$ et al. Assessment of the response in vivo and in vitro of Plasmodium falciparum to sulphadoxinepyrimethamine in the malarious areas of Iran. Journal of tropical medicine and hygiene, 1993, 96:237-40.

33. Koella JC. Costs and benefits of resistance against antimalarial drugs. Parasitology today, 1998, 14:360-4.

34. Delfini LF. The first case of Plasmodium falciparum resistance to chloroquine treatment discovered in the Republic of Afghanistan. Transactions of the Royal Society of Tropical Medicine and Hygiene, 1989, 83(3):316.

35. Rowland $M$ et al. Resistance of falciparum malaria to chloroquine and sulfadoxine-pyrimethamine in Afghan refugee settlements in western Pakistan: surveys by the general health services

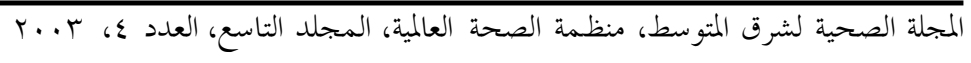


using a simplified in vivo test. Tropical medicine and international health, 1997, 2(11):1049-56.

36. Edrissian $\mathrm{GH}$ et al. Monitoring the response of Plasmodium falciparum and $P$. vivax to antimalarial drugs in the malarious areas in South-East Iran. Archives of Iranian medicine, 1999 2(2):61-6.

37. Report on the intercountry workshop on monitoring therapeutic efficacy of antimalarial drugs. Sana'a, Republic of Yemen, 21-25 April 2002. Cairo, World Health Organization, 2002 (WHO-EM/ $\mathrm{MAL} / 281 / \mathrm{E} / \mathrm{L})$.

38. Edrissian $\mathrm{GH}$, Shahabi S. Preliminary study of the response of Plasmodium falciparum to chloroquine in Sistan and Baluchestan province of Iran. Transactions of the Royal Society of Tropical Medicine and Hygiene, 1985, 79(4): 563-4.

39. Edrissian GH et al. The response of Plasmodium falciparum to chloroquine and mefloquine in Bandar-Abbas and Minab areas, Hormozgan Province, southern Iran. Journal of tropical medicine and hygiene, 1989, 92(2):75-9.

40. Payne D. Practical aspects of the in vivo testing for sensitivity of human Plasmodium spp. to antimalarials. 1982 (unpublished document WHO MAL/82-998, available on request from Communicable Disease Prevention and Control, World Health Organization, 1211 Geneva 27, Switzerland).

41. In vitro micro test (Mark II) for the assessment of the response of Plasmodium falciparum to chloroquine, mefloquine, quinine, sulfadoxine/pyrimethamine and amodiaquine. Geneva, World Health Organization, 1990 (MAP/87.2, Rev 1).

42. Edrissian $\mathrm{GH}$ et al. In vitro response of Plasmodium falciparum to mefloquine in south-eastern Iran. Transactions of the
Royal Society of Tropical Medicine and Hygiene, 1987, 81(1):164-5.

43. Edrissian $\mathrm{GH}$. Status of the response of Plasmodium falciparum to chloroquine and mefloquine in Iran. Tropical and geographical medicine, 1989, 41(4):297303.

44. Zakeri S et al. High prevalence of double Plasmodium falciparum dhfr mutations at codons 108 and 59 in the SistanBaluchistan province, Iran. Journal of infectious diseases, 2003, 187:1828-9.

45. Jafari $S$ et al. Molecular survey of Plasmodium falciparum resistance in southeastern Iran. Annals of tropical medicine and parasitolology, 2003, 97(2):119124.

46. Robinson DS et al. Chloroquine resistant malaria in Pakistan. Lancet, 1984, 2(8409):987.

47. Fox E et al. Chloroquine-resistant Plasmodium falciparum: now in Pakistani Punjab. Lancet, 1985, 1(8443):1432-5.

48. Kinsara AJ et al. Chloroquine-resistant Plasmodium falciparum malaria: report of two locally acquired infections in Saudi Arabia. American journal of tropical medicine and hygiene, 1997, 56: 573-5.

49. Alrajhi AA. Possible establishment of chloroquine-resistant Plasmodium falciparum in Saudi Arabia. American journal of tropical medicine and hygiene, 1999, 60:519-20.

50. Alrajhi AA et al. Chloroquine-resistant Plasmodium falciparum cerebral malaria in a chloroquine-susceptible area. Journal of infectious diseases, 1999, 180:1738-41.

51. al Arishi HM, el Awad Ahmed F, al Bishi LA. Chloroquine-resistant Plasmodium falciparum malaria among children seen in a regional hospital, Tabuk, Saudi Arabia. Transactions of the Royal Society

المجلة الصحية لشرق المتوسط، منظمة الصحة العالمية، المجلد التاسع، العدد ع، ب... 
of Tropical Medicine and Hygiene, 2001, 95:439-40.

52. Malik GM, Khan AM, Abdalla SEA. No response to chloroquine therapy in a case of a Saudi falciparum malaria. Saudi medical journal, 1997, 18:99100.

53. Manohar $\mathrm{S}$ et al. Chloroquine-resistant Plasmodium falciparum malaria in a pregnant woman. Annals of Saudi medicine, 1997, 17:247-9.

54. Malik GM et al. Clinical aspects of malaria in the Asir region, Saudi Arabia. Annals of Saudi medicine, 1998, 18:1517.

55. Ghalib HW et al. Therapeutic efficacy of chloroquine against uncomplicated Plasmodium falciparum malaria in south-western Saudi Arabia. Annals of tropical medicine and parasitology, 2001, 95:773-9.

56. Bayoumi RA et al. Effect of previous chloroquine intake on in vivo $P$. falciparum drug sensitivity. East African medical journal, 1997, 74(5):278-82.

57. Iqbal $\mathrm{J}$ et al. Drug-resistant Plasmodium falciparum infection in immigrants and non-immune travellers. Clinical microbiology and infection, 2002, 8:734-8.

58. Teklehaimanot A. Chloroquine-resistant Plasmodium falciparum malaria in Ethiopia. Lancet, 1986, 2:127-9.

59. Warsame M et al. Susceptibility of Plasmodium falciparum to chloroquine and mefloquine in Somalia. Transactions of the Royal Society of Tropical Medicine and Hygiene, 1988, 82:202-4.

60. Warsame $M$ et al. The changing pattern of Plasmodium falciparum susceptibility to chloroquine but not to mefloquine in a mesoendemic area of Somalia. Transactions of the Royal Society of Tropical Medicine and Hygiene, 1991, 85:200-3.
61. Assessment of therapeutic efficacy of antimalarial drugs for uncomplicated falciparum malaria in areas with intense transmission. Geneva, World Health Organization, 1996. (MAL/96.1077).

62. Warsame M et al. Therapeutic efficacy of chloroquine and sulfadoxine/pyrimethamine against Plasmodium falciparum infection in Somalia. Bulletin of the World Health Organization, 2002, 80:704-8.

63. Warsame M et al. Susceptibility of Plasmodium falciparum to chloroquine and mefloquine in Somalia. Transactions of the Royal Society of Tropical Medicine and Hygiene, 1988, 82:202-4.

64. Smoak BL et al. Plasmodium vivax infections in U.S. Army troops: failure of primaquine to prevent relapse in studies from Somalia. American journal of tropical medicine and hygiene, 1997, 56: 231-4.

65. Warsame M. Establishing sentinel sites for monitoring antimalarial drug resistance in Somalia. Presentation at the Third Inter-Country Meeting of National Malaria Programme Managers, Lahore, Pakistan, 12-15 May 2003. Available at http://www.emro.who.int/rbm/Presentations/PAK03/Tuesday/NationalDrug Policy-SOM.ppt (accessed 19/05/2004).

66. Bruce-Chwatt LJ. Resistance of Plasmodium falciparum to chloroquine in Africa: true or false. Transactions of the Royal Society of Tropical Medicine and Hygiene, 1970, 64:776-84.

67. Kean BH. Chloroquine-resistant falciparum from Africa. Journal of the American Medical Association, 1979, 241(4):395.

68. Omer AHS. Response of Plasmodium falciparum in Sudan to chloroquine. American journal of tropical medicine and hygiene, 1978, 27(5):853-7. 
69. Kouznetsov $\mathrm{RL}$ et al. Use of the in vitro microtechnique for the assessment of drug sensitivity of Plasmodium falciparum in Sennar, Sudan. Bulletin of the World Health Organization, 1980, 58(5): 785-9.

70. Al-Tawil N, Akood MA. Response of falciparum malaria to standard regimen of chloroquine in Khartoum Province, Sudan (unpublished document). WHO/ MAL.83.991 (1983).

71. Al-Tawil N, Akood MA. Response of falciparum malaria to standard regimen of chloroquine in Khartoum Province, Sudan. East African medical journal, 1983, 60:663-8.

72. Lienhardt C, Ghebray R, Candolfi E. Does chloroquine resistance occur in refugee camps in eastern Sudan? Transactions of the Royal Society of Tropical Medicine and Hygiene, 1986, 83:486-7.

73. Abdel-Hameed AA, El-Jak IE, Faragalla IA. Sentinel posts for monitoring therapeutic efficacy of antimalarial drugs against Plasmodium falciparum in the Sudan. African journal of medicine and medical sciences, 2001, 30(suppl.):1-5.

74. Final reports from sentinel sites. National Malaria Control Programme, National Malaria, Schistosomiasis and Leishmaniasis Administration. Khartoum, Federal Ministry of Health, 1997-2002.

75. Medani AR, Younis SA, Saeed NS. Pyrimethamine-sulphadoxine efficacy and prevalence of DHFR point mutations in Plasmodium falciparum field isolates from an area with seasonal transmission in Sudan. In: Moving targets, parasites, resistance and access to drugs. Proceedings of a colloquium. Antwerp, 4-6 December 2000. Tropical medicine and international health, 2001, 6(11):84364.

76. Ibrahim AM, Ali FR, Ali ME. Assessment of chloroquine resistance of Plasmo- dium falciparum in children of Wad Medani (Central Sudan). Journal of tropical pediatrics, 1992, 38(4):162-6.

77. Guthmann JP et al. Field research, relief work and war: does chloroquine-resistance occur in displaced populations of southern Sudan? Tropical doctor, 1996 26:89-90.

78. Elkheir HK et al. Efficacy of suphadoxine and pyrimethamine, doxycycline and their combination in the treatment of resistant falciparum malaria. Saudi medical journal, 2001, 22:690-3.

79. Nour BYM et al. In vitro study to assess the response of Plasmodium falciparum to chloroquine, sulfadoxine-pyrimethamine, quinine and mefloquine in Wad Medani, Sudan 1997/1998. In: Moving targets, parasites, resistance and access to drugs. Proceedings of a colloquium. Antwerp, 4-6 December 2000. Tropical medicine and international health, 2001, 6(11):843-64.

80. Nour BYM. In vitro study to assess the response of Plasmodium falciparum to chloroquine, sulfadoxine-pyrimethamine, quinine and mefloquine in Wad Medani, Sudan [MSc thesis]. University of Gezira, Wad Medani, Sudan, 1999.

81. Babiker $\mathrm{AH}$ et al. Genetic diversity of Plasmodium falciparum in a village in eastern Sudan. 2. Drug resistance, molecular karyotypes and the mdr1 genotype of recent isolates. Transactions of the Royal Society of Tropical Medicine and Hygiene, 1991, 85:578-83.

82. Awad-El-Kariem FM, Miles MA, Warhurst DC. Chloroquine-resistant Plasmodium falciparum isolates from the Sudan lack two mutations in the pfmdr1 gene thought to be associated with chloroquine resistance. Transactions of the Royal Society of Tropical Medicine and Hygiene, 1992, 86:587-9.

المجلة الصحية لشرق المتوسط، منظمة الصحة العالمية، المجلد التاسع، العدد ع، ب... 
83. Bayoumi RAL et al. Drug response and genetic characterization of Plasmodium falciparum clones recently isolated from a Sudanese village. Transactions of the Royal Society of Tropical Medicine and Hygiene, 1993, 87:454-8.

84. Babiker HA et al. High-level chloroquine resistance in Sudanese isolates of Plas- modium falciparum is associated with mutation in the chloroquine resistance transporter gene pfort and the multidrug resistance gene pfmdr1. Journal of infectious diseases, 2001, 183:1535-8.

\section{Improving access to antimalarial medicines. Report of the RBM Partnership Meeting}

Following an evaluation of the Roll Back Malaria (RBM) partnership in 2002, it was suggested that more be done to develop a coherent and coordinated response of partners to the needs of countries in developing and implementing treatment policies. In response, a meeting of the RBM partnership was convened from 30 September to 2 October 2002.

The meeting's overall objective was to define how access to antimalarial drug treatment could be improved in the context of new and challenging treatment policies. It also aimed to create consensus on actions to support implementation of more effective treatment options, guided by country experience and realities.

The specific objectives were: to identify, through country experiences, issues involved in making rational selections of antimalarial treatment policies and their implementation; to identify the role that RBM partners can play in supporting the implementation of policies; to define a coordinated partnership strategy for country support; to review policies for financing antimalarial treatments in endemic countries, with a view to ensuring universal access to treatment.

The report of the meeting can be accessed free online at: http:// whalibdoc.who.int/hq/2003/WHO_CDS_RBM_2003.44.pdf

المجلة الصحية لشرق المتوسط، منظمة الصحة العالمية، المجلد التاسع، العدد ؟، ب... 